\title{
Whole-Genome Sequencing Analysis of Nontyphoidal Salmonella enterica of Chicken Meat and Human Origin Under Surveillance in Sri Lanka
}

\author{
Moon Y.F. Tay, ${ }^{1,2}$ Sujatha Pathirage, ${ }^{3}$ Lakshmi Chandrasekaran,, ${ }^{1,2}$ Uddami Wickramasuriya, ${ }^{3}$ \\ Nirasha Sadeepanie, ${ }^{3}$ Kaushalya D.K. Waidyarathna, ${ }^{3}$ Liyanaralalage Dilini Chathurika Liyanage, ${ }^{3}$ \\ Kelyn L.G. Seow, ${ }^{1,2}$ Rene S. Hendriksen, ${ }^{4}$ Masami T. Takeuchi, ${ }^{5}$ and Joergen Schlundt ${ }^{1,2}$
}

\begin{abstract}
A total of 73 nontyphoidal Salmonella enterica isolates, 33 from raw chicken meat and 40 from routine clinical specimens, were collected between 2015 and 2017 from eight cities in Sri Lanka for a pilot study of whole-genome sequencing for Salmonella surveillance. The isolates were characterized by conventional serotyping and wholegenome sequencing. The raw sequenced data were assembled and analyzed to predict Salmonella serotypes, determine sequence type (ST) profiles of genome and plasmid, and identify plasmid replicon sequences and antimicrobial resistance (AMR) genes. The most common serovar isolated from chicken meat was Salmonella enterica serovar Agona of ST13 $(n=16)$, in contrast to Salmonella enterica serovar Enteritidis of ST11 $(n=21)$ in human. Salmonella enterica serovar Corvallis is the only serovar that was overlapping between human and chicken meat. The level of agreement between serotyping and serotype prediction results was $100 \%$. Among the 33 chicken isolates, multidrug resistance (MDR) was observed in five isolates, including two Salmonella enterica serovar Kentucky ST314, which harbored six different classes of AMR determinants. Among the 40 human isolates, MDR was detected in two Salmonella enterica serovar Chester (ST2063) isolates containing five different antibiotic classes of AMR determinants. Out of 73 isolates, the only human Salmonella enterica serovar Typhimurium strain of ST36 was found to possess extended-spectrum beta-lactamase (ESBL) gene, bla CTX-M-15 $_{15}$, and it was positive for ESBL production. In summary, this study identified S. enterica serovars that were dominating in chicken meat and human and showed the genomic differences among the chicken meat and human strains. It should be noted that the limited number of isolates and sampling at a different time period means that thorough source attribution is not possible. To the best of our knowledge, this is the first report on the use of whole-genome sequencing analysis of nontyphoidal S. enterica isolated from chicken meat and human in Sri Lanka.
\end{abstract}

Keywords: Salmonella enterica, CTX-M-15, whole genome sequencing, Sri Lanka, human, chicken meat, surveillance

\section{Short Report/Case Study}

$S^{n}$ ALMONELLA ENTERICA, a common foodborne pathogen worldwide, has $>2600$ serovars that can cause infections of varying severity to human and animal. The nontyphoidal Salmonella (NTS) strains may be host generalist with broad host specificity that colonizes or infect a wide range of vertebrate animals or may be restricted to particular animal species (Feasey et al., 2012). NTS infections usually cause mild to moderate self-limiting gastroenteritis in young adults, and no antibiotic treatment is required. However, in $\sim 6 \%$ of the gastroenteritis cases, bacteria may proceed to cause an

\footnotetext{
${ }^{1}$ School of Chemical and Biomedical Engineering, Nanyang Technological University (NTU), Singapore, Singapore.

${ }^{2}$ Nanyang Technological University Food Technology Centre (NAFTEC), Nanyang Technological University (NTU), Singapore, Singapore.

${ }^{3}$ Enteric Reference Laboratory, Department of Bacteriology, Medical Research Institute (MRI), Colombo, Sri Lanka.

${ }_{5}^{4}$ National Food Institute, Technical University of Denmark, Kongens Lyngby, 2800, Denmark.

${ }^{5}$ Food and Agriculture Organization of the United Nations (FAO), Rome, Italy.

(C) Moon Y.F. Tay, et al. 2019; Published by Mary Ann Liebert, Inc. This Open Access article is distributed under the terms of the Creative Commons License (http://creativecommons.org/licenses/by/4.0), which permits unrestricted use, distribution, and reproduction in any medium, provided the original work is properly cited.
} 
invasive extraintestinal disease leading to bacteremia and focal infection in the young, elderly, and immunocompromised humans, and ciprofloxacin and extended-spectrum cephalosporin are commonly prescribed to treat such invasive disease (Rowe et al., 1997). Globally, an increasing prevalence of ciprofloxacin and extended-spectrum cephalosporin resistance have been reported in clinical NTS strains (Crump et al., 2015), and it is thought to be associated with the use of fluoroquinolones and beta-lactams as a growth promoter in food-producing animals. NTS is transmitted through animal products (mainly through eggs, meats, and poultry products) and produce contaminated with animal feces and/or human sewage, and contact with animals and animal environment (Crump et al., 2015).

Ministry of Health in Sri Lanka reported an overall decreasing trend in the incidence of dysentery, enteric fever, and food poisoning for the period of 2007-2017 (MoH, 2018). The number of aforementioned foodborne illness cases that can be attributed to S. enterica in Sri Lanka is unknown. Similarly, the transmission pattern of S. enterica in Sri Lanka remains unclear, and there is no published data characterizing the molecular epidemiology of S. enterica in human and poultry production. In Sri Lanka, both fluoroquinolones and beta-lactams are used to treat human Salmonella infection and are banned for growth promotion purposes (personal communication from Dr. Palika Fernando, National AMR steering committee member, Head Department of Bacteriology, Veterinary Research Institute, Sri Lanka). Given the public health significance of Salmonella, this pilot cross-sectional genomic-based surveillance study is done to provide the NTS situation in humans and raw chicken meats from eight cities in Sri Lanka. It is important to note that this study is not designed to compare the prevalence between the different cities. On the contrary, this study aims to provide a molecular snapshot of genetic variability among the collected Salmonella strains.

A total of 73 nontyphoidal S. enterica isolates, 33 from raw chicken meat and 40 from clinical specimens (i.e., stool, blood, and joint fluid) were collected from eight cities in Sri Lanka, namely Awissawella, Badulla, Colombo, Galle, Jaffna, Kandy, Peradeniya, and Ragama between 2015 and 2017 (Table 1). Genomic DNA extraction, library construction, and sequencing were performed as previously described (Guo et al., 2019). Sequence data were deposited into GenBank under BioProject accession number PRJNA504925. GenBank accession numbers for individual isolates are listed in Table 1. De novo assembly of draft genome (Afgan et al., 2018), assessment of draft genome assembly quality (Gurevich et al., 2013), and genomic analyses (Larsen et al., 2012; Zankari et al., 2012; Carattoli et al., 2014; Zhang et al., 2015; Alikhan et al., 2018) were performed as previously described (Tay et al., 2019). Conventional serotyping according to Kauffman-White scheme was done in-house at the Enteric Reference Laboratory in Medical Research Institute with antisera purchased from S\&A Reagents Lab Ltd., Part. (Thailand).

Genomic analyses showed that Salmonella enterica serovar Agona of ST13 $(n=16)$ and Salmonella enterica serovar Enteritidis of ST11 $(n=21)$ were the most prevalent serovars that were observed among chicken meat and human isolates, respectively. Salmonella enterica serovar Corvallis ST1541 is the only serovar that was overlapping between human and chicken meat in this study. There was $100 \%$ concordance between conventional serotyping by Kauffman-White scheme and genotypic serotype prediction by SeqSero (Zhang et al., 2015). Discrepancy was observed for four human isolates; they were serotyped to be Paratyphi B var java (henceforth Java) but were predicted to be Paratyphi B. They have identical serological formula and Java is considered a variant of Paratyphi B that can ferment d-tartrate, whereas Paratyphi B cannot due to a single nucleotide change in the start codon of the STM3356 gene (Malorny et al., 2003). Hence, when the draft genome of these four isolates were blasted against the STM3356 gene of Java strain NCTC5706 (GenBank accession number: LT571437.1), the start codon was ATG (data not shown). In addition, these isolates were phenotypically tested to be positive for dtartrate fermentation (data not shown). Altogether, this indicates the isolates are able to ferment d-tartrate and they are indeed Paratyphi B var. Java, which tallies with the serotyping result. Hence, this suggests that additional genetic loci or alleles should be taken into consideration for prediction of a certain serotype from sequence data. More than twothirds $(50 / 73)$ of the isolates contained plasmid replicons. The commonly seen plasmid replicons were IncFII(S) and IncFIB(S), of sequence type [F-:A16:B22] and were found in 16 Salmonella Enteritidis strains. Among the chicken meat isolates, $87.9 \%(29 / 33)$ of them had at least one resistance gene and multidrug resistance (MDR; defined as resistance to three or more classes of antibiotics) was observed in $15.2 \%$ of them (5/33), including two Salmonella enterica serovar Kentucky ST314 strains, which harbored six different classes of antimicrobial resistance (AMR) determinants. In contrast, among the human isolates, $17.5 \%$ (7/40) of them had at least one resistance gene and only two isolates $(5 \%, 2 / 40)$ were found to be MDR, which were both Salmonella enterica serovar Chester ST2063 strain that contained five AMR determinants, belonging to five different antibiotic classes. It is worth mentioning that out of 73 isolates, only one human isolate contained extended-spectrum beta-lactamase (ESBL) gene, bla ${ }_{\mathrm{CTX}-\mathrm{M}-15}$. As expected, when we performed the double-disk synergy test (Guo et al., 2019), the strain was tested to be positive for ESBL production. Among all the identified AMR genes, the most frequent resistance genotype was fosA7 and was found in all 16 Salmonella Agona (ST13) strains from different chicken meat samples. We did not test the phenotypic resistance of these isolates to fosfomycin, and hence we do not know if fosA7 gene confers phenotypic resistance to fosfomycin. When a whole-genome single nucleotide polymorphism (SNP) analysis with CFSAN SNP Pipeline (Davis et al., 2015) that was installed on GalaxyTrakr (https://www.galaxytrakr.org) (Afgan et al., 2018) was performed on the 16 Salmonella Agona isolates, the minimum and maximum SNP differences were 0 and 36, respectively (data not shown). Upon construction of the bestscoring maximum likelihood (ML) SNP tree with randomized axelerated ML (RAxML) using a GTRGAMMA model of evolution and default parameters (Stamatakis, 2014), it appears that some isolates are phylogenetically related due to 0 SNP difference, but they may not be epidemiologically related due to lack of information on sampling source.

The investigation has identified the $S$. enterica serovars that were dominating in chicken meat and human, and showed the genomics differences among the chicken meat 


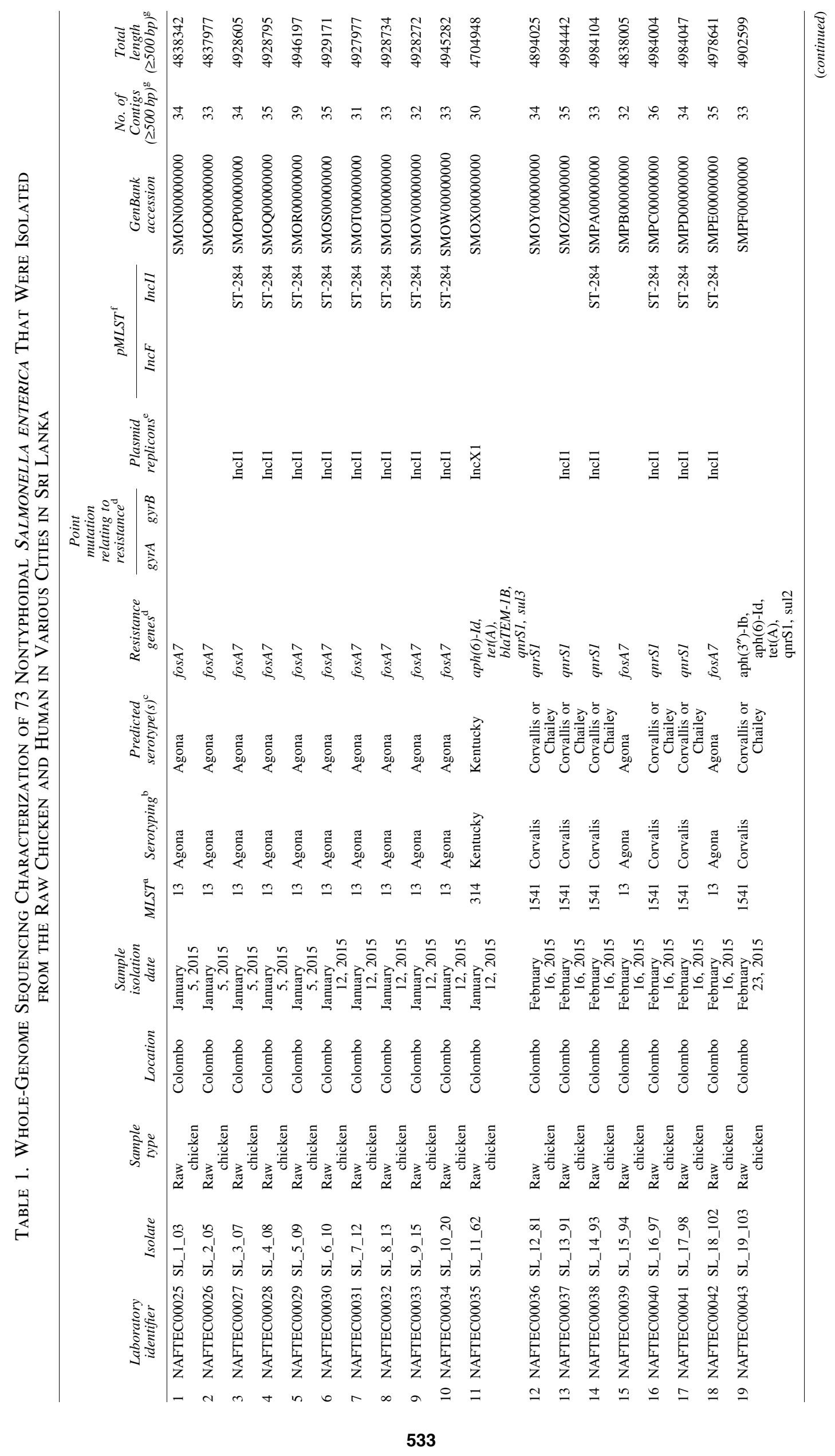




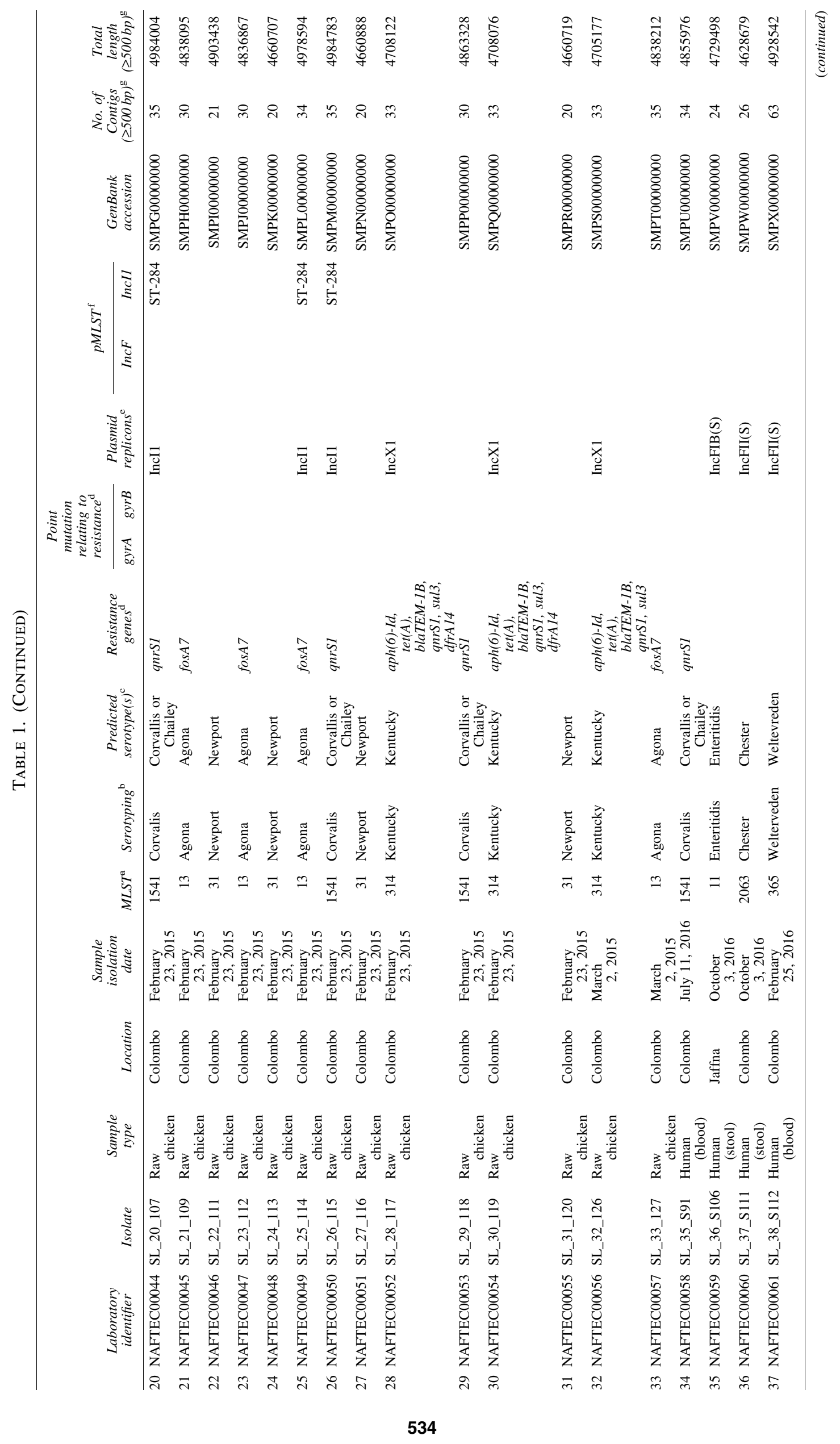




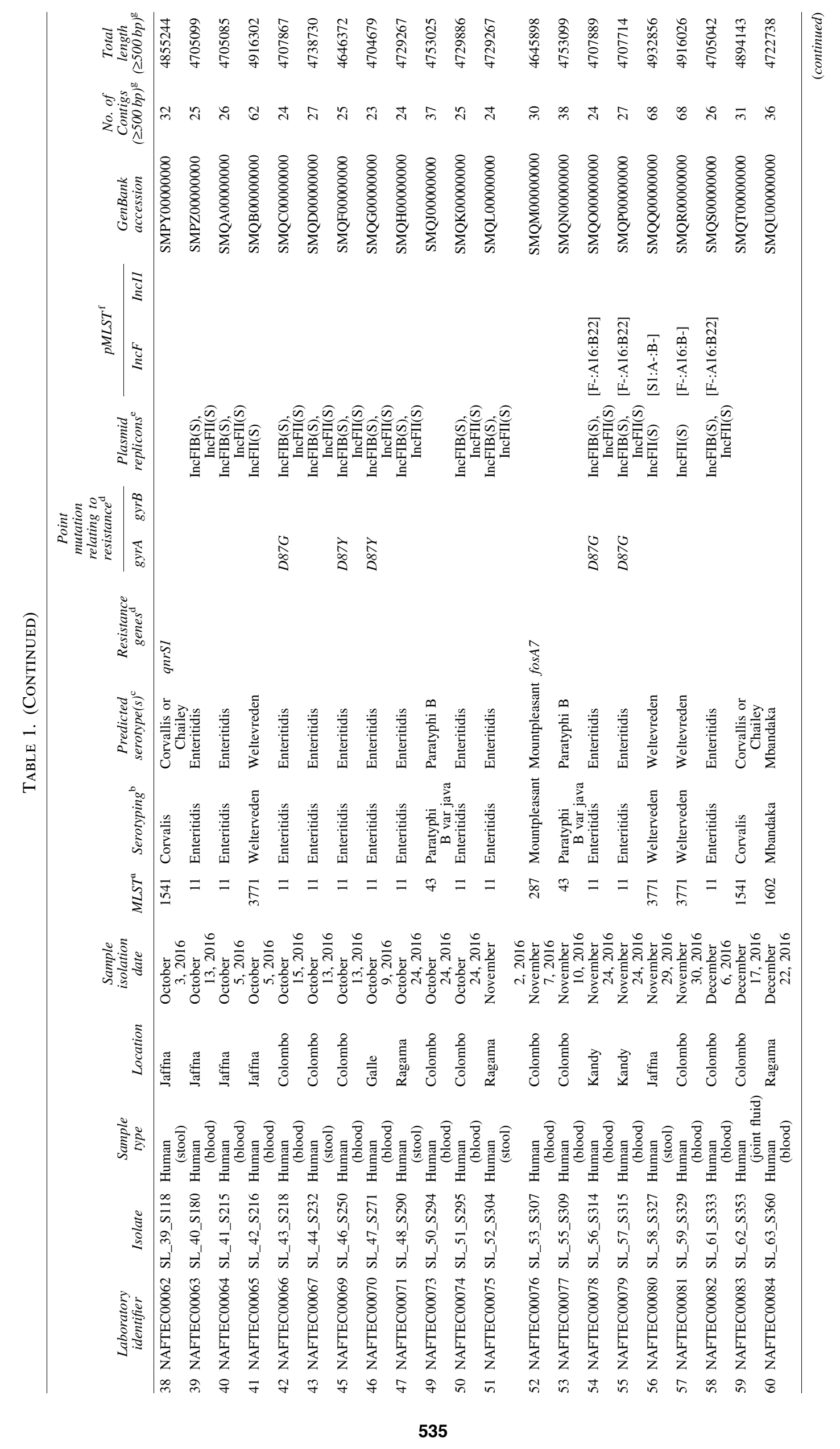




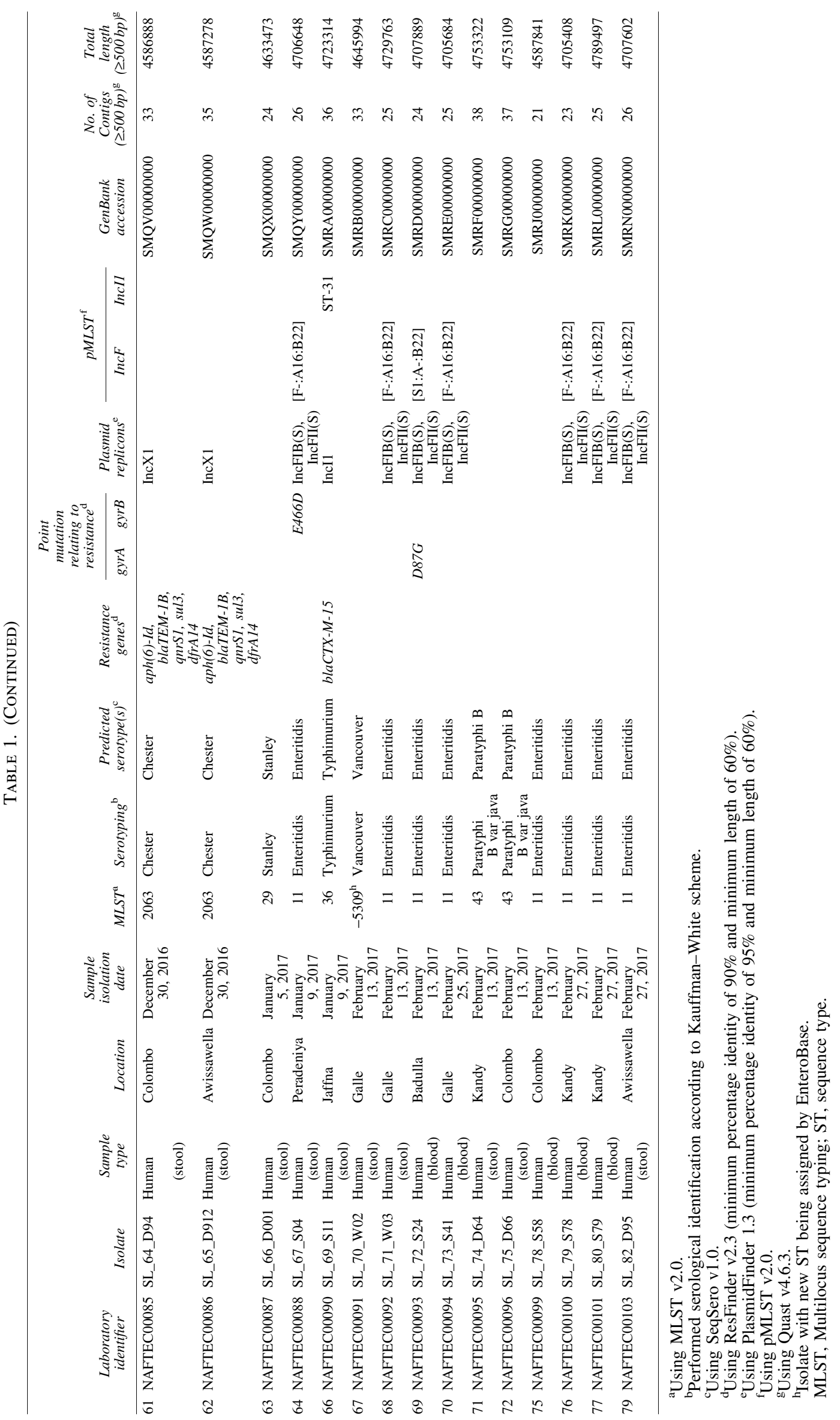


and human strains. Since it is a retrospective study, it is limited by the absence of adequate $(\geq 50)$ and regular sampling at indicated cities/locations for a longer period of time ( $\geq 1$ year), within the same time period for both chicken meat and human samples. Hence, it is not possible to draw any conclusion about the correlation between the clinical isolates and the chicken reservoir. Nevertheless, the generated data do provide very rough details about Salmonella serotypes and resistance traits in chicken meat and human in studied cites, and contribute to the design of sampling framework for prospective Salmonella and AMR surveillance.

\section{Acknowledgments}

This study was supported by funding from Nanyang Technological University (NTU) Research Initiative and conducted under the joint project of NTU Food Technology Centre (NAFTEC) and Food and Agriculture Organization of the United Nations (FAO), entitled "Towards better food safety management through the use of Next Generation Sequencing for foodborne pathogenic and antimicrobial resistance bacteria in a One Health context."

\section{Authors' Contributions}

M.Y.F.T. performed bioinformatics analysis, analyzed and interpreted the data, and drafted and coordinated the article writing. S.P. analyzed and interpreted the data, and contributed to article writing. L.C. performed bioinformatics analysis. U.W. did meat sampling and performed bacterial isolation and identification. N.S., K.D.K.W., and L.R.D.C.L. worked on the human isolates. K.L.G.S. performed DNA extraction. R.S.H., M.T.T., and J.S. contributed to article writing and provided scientific advice.

\section{Disclosure Statement}

No competing financial interests exist.

\section{References}

Afgan E, Baker D, Batut B, et al. The Galaxy platform for accessible, reproducible and collaborative biomedical analyses: 2018 update. Nucleic Acids Res 2018;46:W537-W544.

Alikhan NF, Zhou Z, Sergeant MJ, Achtman M. A genomic overview of the population structure of Salmonella. PLoS Genet 2018;14:e1007261.

Carattoli A, Zankari E, Garcia-Fernandez A, et al. In silico detection and typing of plasmids using PlasmidFinder and plasmid multilocus sequence typing. Antimicro Agents Chemother 2014;58:3895-3903.

Crump JA, Sjolund-Karlsson M, Gordon MA, Parry CM. Epidemiology, clinical presentation, laboratory diagnosis, antimicrobial resistance, and antimicrobial management of invasive Salmonella infections. Clin Microbiol Rev 2015;28:901-937.

Davis S, Pettengill JB, Luo Y, et al. CFSAN SNP Pipeline: An automated method for constructing SNP matrices from next-generation sequence data. PeerJ Comput Sci 2015;1: e20.
Feasey NA, Dougan G, Kingsley RA, Heyderman RS, Gordon MA. Invasive non-typhoidal salmonella disease: An emerging and neglected tropical disease in Africa. Lancet 2012; 379:2489-2499.

Guo S, Tay MYF, Aung KT, et al. Phenotypic and genotypic characterization of antimicrobial resistant Escherichia coli isolated from ready-to-eat food in Singapore using disk diffusion, broth microdilution and whole genome sequencing methods. Food Control 2019;99:89-97.

Gurevich A, Saveliev V, Vyahhi N, Tesler G. QUAST: Quality assessment tool for genome assemblies. Bioinformatics 2013; 29:1072-1075.

Larsen MV, Cosentino S, Rasmussen S, et al. Multilocus sequence typing of total-genome-sequenced bacteria. J Clin Microbiol 2012;50:1355-1361.

Malorny B, Bunge C, Helmuth R. Discrimination of d-tartratefermenting and -nonfermenting Salmonella enterica subsp. enterica isolates by genotypic and phenotypic methods. J Clin Microbiol 2003;41:4292-4297.

Ministry of Health. Weekly Epidemiological Report. Volume 2018. Sri Lanka: Epidemiology Unit, Ministry of Health, 2018.

Rowe B, Ward LR, Threlfall EJ. Multidrug-resistant Salmonella typhi: A worldwide epidemic. Clin Infect Dis 1997;24 Suppl 1:S106-S109.

Stamatakis A. RAxML version 8: A tool for phylogenetic analysis and post-analysis of large phylogenies. Bioinformatics 2014;30:1312-1313.

Tay MYF, Adzitey F, Sultan SA, Tati JM, Seow KLG, and Schlundt J. Whole-genome sequencing of nontyphoidal Salmonella enterica isolates obtained from various meat types in Ghana. Microbiol Res Anncmnts 2019;8.

Zankari E, Hasman $\mathrm{H}$, Cosentino S, et al. Identification of acquired antimicrobial resistance genes. J Antimicrob Chemother 2012;67:2640-2644.

Zhang S, Yin Y, Jones MB, et al. Salmonella serotype determination utilizing high-throughput genome sequencing data. J Clin Microbiol 2015;53:1685-1692.

Address correspondence to: Moon Y.F. Tay, PhD

School of Chemical and Biomedical Engineering Nanyang Technological University (NTU) 62 Nanyang Drive Singapore 637459 Singapore

E-mail: moon.tay@ntu.edu.sg

Sujatha Pathirage, MD Medical Research Institute (MRI) PO Box 527

Dr. Danister Silva Mawatha Colombo 00800 Sri Lanka

E-mail: chansujat@yahoo.com 